\title{
Registration and Fusion of Blurred Images
}

\author{
Filip Sroubek and Jan Flusser \\ Institute of Information Theory and Automation \\ Academy of Sciences of the Czech Republic \\ Pod vodárenskou věží 4, 182 08, Praha 8 \\ \{sroubekf, fluser\}@utia.cas.cz
}

\begin{abstract}
We present a maximum a posteriori solution to problems of accurate registration of blurred images and recovery of an original undegraded image. Our algorithm has the advantage that both tasks are performed simultaneously. An efficient implementation scheme of alternating minimizations is presented. A simulation and a real-data experiment demonstrate the superb performance of the algorithm.
\end{abstract}

\section{Introduction}

Imaging sensors and other devices have their physical limits and imperfections, therefore, an acquired image represents only a degraded version of the original scene. Two main categories of degradations are recognized: color (or brightness) degradations and geometric degradations. The former degradations are caused by such factors as wrong focus, motion of the scene, media turbulence, noise, and limited spatial and spectral resolution of the sensor; they usually result in image blurring. The latter degradations originate from the fact that each image is a $2-\mathrm{D}$ projection of $3-\mathrm{D}$ world. They cause deformations of object shapes and other spatial distortions of the image.

Since the geometric and color degradations are in principle inevitable in real applications, analysis and interpretation of degraded images represents the key problem. Image fusion provides a solution to this problem and consists of two steps. First the geometric deformations are removed by means of image registration, and second, the color (intensity) information is combined. If we can model the color deformation by convolution, the second step corresponds to a multichannel blind deconvolution (MBD) problem. In this paper, we address the problem of registration of blurred images (channels) from the perspective of image fusion.

Image registration is a process of transforming two or more images into a geometrically equivalent form. It eliminates degradation effects caused by the geometric distortion. For images which are not blurred, the registration has been extensively studied in the recent literature (see [1] for a survey). However, blurred images require special registration techniques. They can be, as well as the general-purpose registration methods, divided into two groups - global and landmark-based techniques. Regardless of the particular technique, all feature 
extraction methods, similarity measures, and matching algorithms used in the registration process must be insensitive to image blurring.

Global methods do not search for particular landmarks in the images. They try to estimate directly the between-channel translation and rotation. Myles and Lobo [2] proposed an iterative method working well if a good initial estimate of transformation parameters is available. Zhang et al. [3] proposed to estimate the registration parameters by bringing the channels into a canonical form. Since blur-invariant moments were used to define the normalization constraints, neither the type nor the level of the blur influences the parameter estimation. Kubota et al. [4] proposed a two-stage registration method based on hierarchical matching, where the amount of blur is considered as another parameter of the search space. Zhang and Blum [5] proposed iterative multiscale registration based on optical flow estimation in each scale, claiming that optical flow estimation is robust to image blurring. All global methods require a considerable (or even complete) spatial overlap of the channels to yield reliable results, which is their major drawback.

Landmark-based blur-invariant registration methods have appeared very recently, just after the first paper on the moment-based blur-invariant features [6]. Originally, these features could only be used for registration of mutually shifted images. The proposal of their rotational-invariant version [7] in combination with a robust detector of salient points [8] led to registration methods that are able to handle blurred, shifted and rotated images [9].

Although the above-cited registration methods are very sophisticated and can be applied almost to all types of images, the result tends to be rarely perfect. The registration error is usually few pixels for blurred images. However, the second step of image fusion (MBD in our case) requires perfectly aligned channels. Current MBD methods, see e.g. [10-13], are not sufficiently robust to handle the registration error. In the field of super-resolution image reconstruction, a few techniques, such as [14], were proposed that simultaneously estimate motion vectors and reconstruct the image, but they are not blind or assume the same parametrized blur in the channels. The first step towards more robust MBD was given in [15], which deals with blind deconvolution of translated channels degraded by different but simple motion blurs.

In this paper, we propose a novel technique that can be applied after removing large between-channel misregistrations and which then performs image fusion in one step, i.e. fine registration and blind deconvolution simultaneously. Image blurring can be arbitrary and is unknown, while the geometric misregistrations are supposed to be also unknown but limited to "small" translations. In the next section, we formulate the solution as a maximum a posteriori estimator (MAP) and use an alternating minimization (AM) algorithm to find the solution. We derive a priori probabilities of the original image and the blurs from properties of bounded variation functions and the multichannel framework, respectively. Experimental results are given in Section 3. Finally, Section 4 concludes the paper. 


\section{MAP analysis}

Let us assume that the $k$-th acquired image (channel) $z_{k}$ can be modelled by blurring the "ideal" image $u$ and shifting the result by few pixels

$$
z_{k}\left(x+a_{k}, y+b_{k}\right)=\left(u * h_{k}\right)(x, y)+n_{k}(x, y)
$$

where vector $\left(a_{k}, b_{k}\right)=t_{k}$ represents the unknown translation of the $k$-th channel, $h_{k}$ is the unknown blur mask with a characteristic of a low-pass filter, and $n_{k}$ denotes additive noise. In the discrete domain, this degradation model takes the form:

$$
\mathbf{z}_{k}=\mathbf{T}_{k} \mathbf{H}_{k} \mathbf{u}+\mathbf{n}_{k}, k=1, \ldots, K,
$$

where $\mathbf{z}_{k}, \mathbf{u}$, and $\mathbf{n}_{k}$ are discrete lexicographically ordered equivalents of the image functions $z_{k}, u$, and $n_{k}$, respectively. $\mathbf{T}_{k}$ is a translation operator shifting the image by $t_{k}$ pixels, i.e. a linear filter with the delta function at the position $t_{k}$. One can readily see that the matrix product $\mathbf{T}_{k} \mathbf{H}_{k}=\mathbf{G}_{k}$ defines convolution with a mask $\mathbf{g}_{k}$ that is a shifted version of a mask $\mathbf{h}_{k}$ (discrete representation of $\left.h_{k}\right)$. This degradation model closely resembles the model used in super resolution except that a subsampling operator is not present in our case. By concatenating the channels, the previous equation can be rewritten in two equivalent forms

$$
\mathbf{z}=\mathbf{G u}+\mathbf{n}=\mathbf{U g}+\mathbf{n}
$$

where $\mathbf{z} \equiv\left[\mathbf{z}_{1}^{T}, \ldots, \mathbf{z}_{K}^{T}\right]^{T}, \mathbf{G} \equiv\left[\mathbf{G}_{1}^{T}, \ldots, \mathbf{G}_{K}^{T}\right]^{T}, \mathbf{n} \equiv\left[\mathbf{n}_{1}^{T}, \ldots, \mathbf{n}_{K}^{T}\right]^{T}$, $\mathbf{g} \equiv\left[\mathbf{g}_{1}^{T}, \ldots, \mathbf{g}_{K}^{T}\right]^{T}$, and $\mathbf{U}$ is a block-diagonal matrix with $K$ blocks each performing convolution with the image $\mathbf{u}$.

We adopt a stochastic approach and follow the MAP formulation proposed in our previous work [16]. The conditional pdf $p(\mathbf{z} \mid \mathbf{u}, \mathbf{g})$ follows from (2) and from our assumption of white Gaussian noise, i.e.

$$
p(\mathbf{z} \mid \mathbf{u}, \mathbf{g}) \propto \exp \left\{-\frac{1}{2}(\mathbf{z}-\mathbf{G u})^{T} \Sigma^{-1}(\mathbf{z}-\mathbf{G u})\right\}
$$

where $\Sigma$ is the noise diagonal covariance matrix with $\left\{\sigma_{k}^{2}\right\}_{k=1}^{K}$ on the corresponding positions on the main diagonal. If the same noise variance $\sigma^{2}$ is assumed in each channel, $\Sigma^{-1}$ reduces to a scalar $\sigma^{-2}$.

A general model for the prior distribution $p(u)$ is a Markov random field which is characterized by its Gibbs distribution given by $p(u) \propto \exp (-F(u) / \lambda)$, where $\lambda$ is a constant and $F$ is called the energy function. One can find various forms of the energy function in the literature, however, the most promising results have been achieved for variational integrals. The energy function then takes the form

$$
F(u)=\int \phi(|\nabla u|),
$$

where $\phi$ is strictly convex, nondecreasing function that grows at most linearly. Examples of $\phi(s)$ are $s$ (total variation), $\sqrt{1+s^{2}}-1$ (hypersurface minimal 
function) or $\log (\cosh (s))$. The energy function based on the variational integral is highly nonlinear and to overcome this difficulty we follow a half-quadratic scheme described in [17]. In addition, we confine the distribution to an amplitude constraint set $C_{u} \equiv\{u \mid \alpha \leq u \leq \beta\}$ with amplitude bounds derived from the input images, typically $\alpha=0$ and $\beta=255$. The prior distribution then takes the form

$$
p(\mathbf{u})= \begin{cases}\frac{1}{Z} \exp \left\{-\frac{1}{2 \sigma_{u}^{2}} \mathbf{u}^{T} \mathbf{L}(v) \mathbf{u}\right\} & \text { if } \mathbf{u} \in C_{u} \\ 0 & \text { otherwise }\end{cases}
$$

where $Z$ is the partition function, $\sigma_{u}^{2}$ denotes the image variance, $\mathbf{u}^{T} \mathbf{L}(v) \mathbf{u}$ represents the discretization of (3) and $v$ is the auxiliary variable introduced by the half-quadratic scheme, which is calculated as $v(x, y)=\phi^{\prime}(|\nabla u(x, y)|) /|\nabla u(x, y)|$.

The shape of the prior distribution $p(\mathbf{g})$ can be derived from a fundamental multichannel constraint stated in [10]. Let $\mathbf{Z}_{k}$ denote the convolution matrix with the degraded image $\mathbf{z}_{k}$. If noise $\mathbf{n}_{k}$ is zero and the original channel masks $\left\{\mathbf{h}_{k}\right\}$ are weakly coprime, i.e. their only common factor is a scalar, then the blurs $\left\{\mathbf{g}_{k}\right\}$ satisfy

$$
\mathbf{Z}_{i} \mathbf{g}_{j}-\mathbf{Z}_{j} \mathbf{g}_{i}=\mathbf{0}, \quad 1 \leq i<j \leq K
$$

provided that the blur size is large enough to accommodate the maximum original blur size and the maximum shift between the channels. After stacking the system of equations (4), we obtain

$$
\mathcal{Z} \mathbf{g}=\mathbf{0}
$$

where $\mathbf{g} \equiv\left[\mathbf{g}_{1}^{T}, \ldots, \mathbf{g}_{K}^{T}\right]^{T}$. If the noise term $\mathbf{n}_{k}$ is present, it follows from (2) that the left-hand side of $(5)$ equals a realization of the Gaussian process of zero mean and covariance $\mathcal{C}=\mathcal{G} \Sigma \mathcal{G}^{T}$, where $\mathcal{G}$ has the form of $\mathcal{Z}$ in (5) with $\mathbf{Z}_{i}$ replaced by $\mathbf{G}_{i}$.

It is desirable to include also other prior knowledge about the blurs, such as positivity or constant energy. We therefore define a set of admissible solutions as $C_{g} \equiv\left\{\mathbf{g} \mid g_{k}(x, y) \geq 0 \wedge \sum_{x, y} g_{k}(x, y)=1, k=1, \ldots, K\right\}$ and propose the following prior distribution:

$$
p(\mathbf{g})= \begin{cases}\frac{1}{Z} \exp \left\{-\frac{1}{2} \mathbf{g}^{T} \mathcal{Z}^{T} \mathcal{C}^{-1} \mathcal{Z} \mathbf{g}\right\} & \text { if } \mathbf{g} \in C_{g} \\ 0 & \text { otherwise }\end{cases}
$$

The matrix $\mathcal{C}$ is constructed by the blurs $\mathbf{g}$, that are to be estimated, and its inversion is not trivial. To overcome this difficulty, we approximate $\mathcal{C}$ by a diagonal matrix $\mathcal{D}$ such that $\operatorname{diag}(\mathcal{D})=\operatorname{diag}(\mathcal{C})$, where $\operatorname{diag}(\cdot)$ denotes the main diagonal of a matrix. The elements of $\mathcal{D}$ take the form $\sigma_{i}^{2}\left\|\mathbf{g}_{j}\right\|^{2}+\sigma_{j}^{2}\left\|\mathbf{g}_{i}\right\|^{2}$ for $1 \leq i<j \leq K$. The value of $\left\|\mathbf{g}_{i}\right\|^{2}$ is not known in advance, but a good initial approximation can be calculated. Since $\mathbf{g} \in C_{g}, \frac{1}{\prod S_{g}} \leq\left\|\mathbf{g}_{i}\right\|^{2} \leq 1$ and we use the bottom limit for $\left\|\mathbf{g}_{i}\right\|^{2}$. 
The MAP estimation is then given by

$$
\{\hat{\mathbf{u}}, \hat{\mathbf{g}}\}=\arg \min _{\substack{\mathbf{u} \in C_{u} \\ \mathbf{g} \in C_{g}}}\left\{(\mathbf{z}-\mathbf{G u})^{T} \Sigma^{-1}(\mathbf{z}-\mathbf{G u})+\frac{1}{\sigma_{u}^{2}} \mathbf{u}^{T} \mathbf{L}(v) \mathbf{u}+\mathbf{g}^{T} \mathcal{Z}^{T} \mathcal{D}^{-1} \mathcal{Z} \mathbf{g}\right\}
$$

Such problems can be solved by means of genetic algorithms or simulated annealing. In this paper we have adopted an approach of alternating minimizations (AM-MAP) over $\mathbf{u}$ and $\mathbf{g}$. The advantage of this scheme lies in its simplicity. Each term in (6) is convex, the derivatives w.r.t. $\mathbf{u}$ and $\mathbf{g}$ can be easily calculated and we alternate between two simple linear equations.

One must supply the blur size to the algorithm. An important feature, which makes the proposed method particulary appealing, is that an accurate estimation is not necessary; we must only guarantee that the blur size is not underestimated. In addition, the noise covariance $\Sigma$ and the image variance $\sigma_{u}^{2}$ are obligatory in the algorithm. If noise has the same variance $\sigma_{n}^{2}$ in every channel, the MAP expression (6) is simplified and only the signal to noise ratio $\sigma_{u}^{2} / \sigma_{n}^{2}$ is required in the algorithm.

\section{$3 \quad$ Experimental results}

To illustrate the performance of the AM-MAP registration and fusion method, we conducted two experiments: one with synthetic data and one with real data.

In the first experiment, we created two degraded images depicted in Fig. 1(a). This was done by convolving the original image with two different $5 \times 5$ blurs, adding noise of $\mathrm{SNR}=50 \mathrm{~dB}$ and shifting one of the blurred images by $5 \times 5$ pixels. The AM-MAP algorithm was initialized with the overestimated blur size $12 \times 12$. The fused image and the estimated blur masks are shown in Fig. 1(b). Reconstructed blurs are properly shifted and de facto perform registration. The fused image is by visual comparison much sharper than the input channels and no artifacts are visible.

In real applications one cannot expect that translation is the only geometric deformation that may occur. As mentioned in the introduction, the registration methods suitable for blurred images must be first applied in such cases. However, precise registration is seldom achieved. If our fusion algorithm is used, one can reduce the effort to reach accurate registration. Fast registration that removes only the major geometric degradation will suffice. To test this issue and applicability of the proposed algorithm, we conducted the experiment with real data.

We photographed a house facade (relatively planar scene) under low-light conditions with a standard $3 \mathrm{Mp}$ digital camera. Three photos (see Fig. 2) were taken from the same spot with the camera held in hand and set to ISO 100. The first two have a long exposure time of $1 / 4 \mathrm{~s}$ which was necessary for the given light conditions. Both images exhibit strong blurring due to the hand motion. We slightly tilted the camera before the second image was taken. The third photo was 


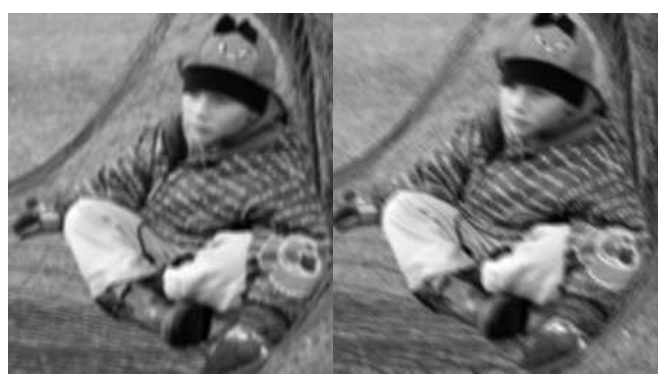

(a)

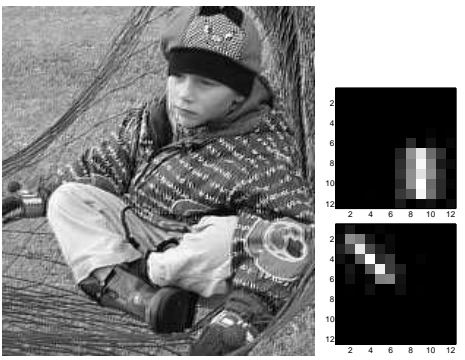

(b)

Fig. 1. AM-MAP image fusion: (a) two blurred and mutually shifted images of size $230 \times 260$ pixels; (b) fused image and estimated translated blur masks.

acquired with a shorter exposure time of $1 / 100$ s to avoid the blurring effect but at the cost of low contrast. Due to the finite quantization step of the digital camera, some details in the image (compare for example sphinx's face) are irrecoverably lost. This image was used only for comparison purposes. Image fusion proceeded as follows. The two degraded images in Figs. 2(a) and 2(b) were first registered with a landmark-based method. Rough registration was performed by means of three control points and an affine mapping function. We could do better and decrease, but never completely cancel, a relatively large registration error $(\approx 10$ pixels $)$, if more control points and/or a more complex mapping were used. Since the goal was to test the robustness of our method, inaccurate registration was sufficient. The roughly registered images then served as inputs to the AMMAP fusion algorithm. We estimated the blur size to be $15 \times 15$ pixels, which was done by first inspecting the blurred letters (see Fig. 3) in the photos and then multiplying the size by two to accommodate possible misregistration. The algorithm was restarted with different signal to noise ratios and provided the best results according to our visual assessment for $\sigma_{u}^{2} / \sigma_{n}^{2}=2000$, which corresponds to SNR between 30 and $40 \mathrm{~dB}$. The fused image and the estimated blurs after 20 iterations are shown in Figs. 2(d). On closer inspection, details in Fig. 3 reveal that the fused image is not as sharp as the short-exposure photo but the overall impression of the image is very pleasing.

\section{Conclusions}

We have developed an iterative fusion algorithm that performs simultaneously registration and restoration of translated blurred images. The fusion problem is formulated as the MAP estimation with the prior probabilities derived from the variational integral and from the mutual relation of coprime channels. We envisage that possible future extensions of the proposed method are to include space-variant deconvolution (the blur mask may depend on the position in the image) and resolution enhancement of the fused image (super-resolution). 


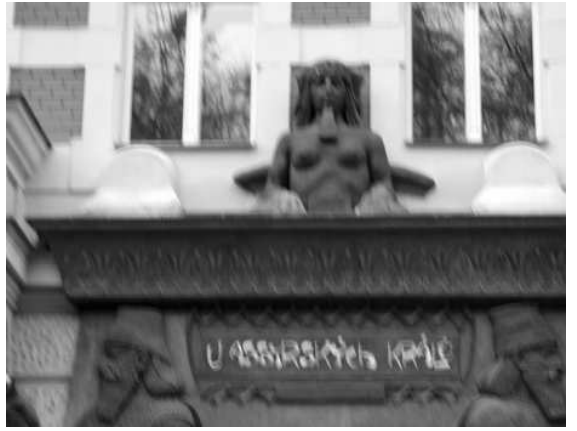

(a)

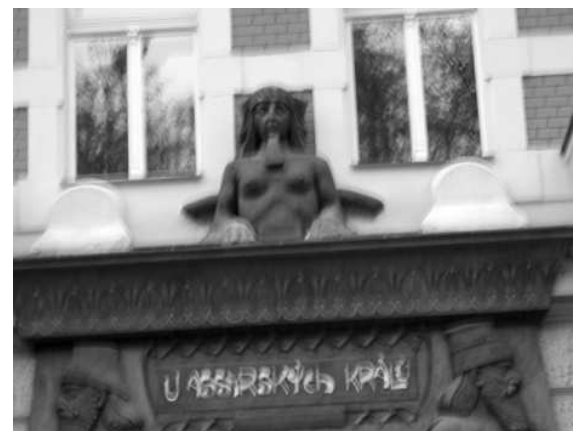

(b)

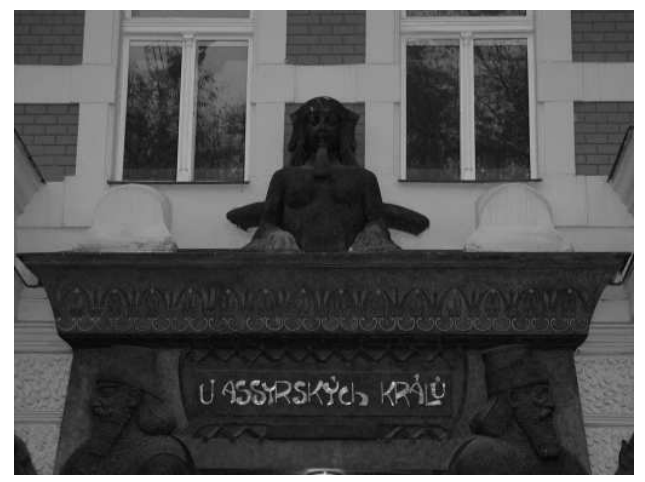

(c)

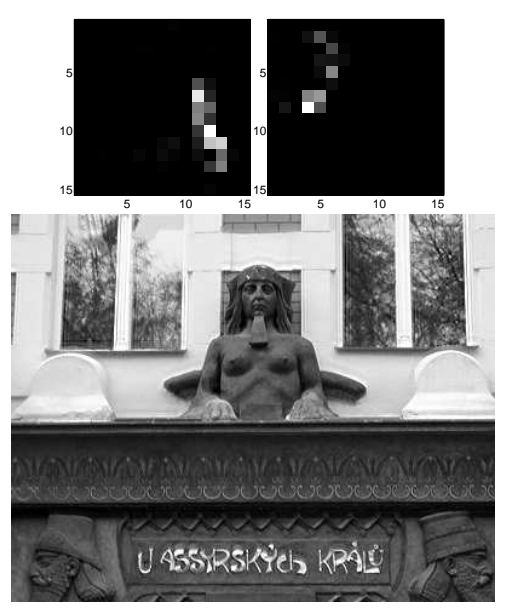

(d)

Fig. 2. Real data experiment: A house facade was photographed with a standard digital camera under low-light conditions. All photos were taken with the hand-held camera and downscaled by four. (a)-(b) Two images acquired with a correct but long exposure time of $1 / 4 \mathrm{~s}$. The images exhibit high contrast but severe blurring is evident due to the hand motion. (c) Image acquired with a short exposure time of $1 / 100 \mathrm{~s}$. This image is sharp but too dark. The two top images were roughly registered and then fused with the AM-MAP algorithm. (d) Result of image fusion.

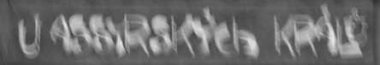

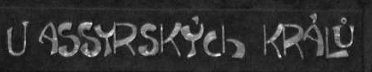

U ASSYRSKYCL KRAOÜ

Fig. 3. Close-ups: (top) detail of the blurred image in Fig. 2(a); (middle) detail of the sharp image in Fig. 2(c); (bottom) detail of the fused image in Fig. 2(d). 


\section{Acknowledgments}

Financial support of this research was provided mainly by the Grant Agency of the Czech Republic under the project No. 102/04/0155 and partially by the bilateral project 2003CZ0009 CSIC-Academy of Sciences of the Czech Republic.

\section{References}

1. Zitová, B., Flusser, J.: Image registration methods: A survey. Image and Vision Computing 21 (2003) 977-1000

2. Myles, Z., Lobo, N.V.: Recovering affine motion and defocus blur simultaneously. IEEE Trans. Pattern Analysis and Machine Intelligence 20 (1998) 652-658

3. Zhang, Y., Wen, C., Zhang, Y., Soh, Y.C.: Determination of blur and affine combined invariants by normalization. Pattern Recognition 35 (2002) 211-221

4. Kubota, A., Kodama, K., Aizawa, K.: Registration and blur estimation methods for multiple differently focused images. In: Proceedings International Conference on Image Processing. Volume II. (1999) 447-451

5. Zhang, Z., Blum, R.: A hybrid image registration technique for a digital camera image fusion application. Information Fusion 2 (2001) 135-149

6. Flusser, J., Suk, T., Saic, S.: Recognition of blurred images by the method of moments. IEEE Trans. Image Processing 5 (1996) 533-538

7. Flusser, J., Zitová, B.: Combined invariants to linear filtering and rotation. Intl. J. Pattern Recognition Art. Intell. 13 (1999) 1123-1136

8. Zitová, B., Kautsky, J., Peters, G., Flusser, J.: Robust detection of significant points in multiframe images. Pattern Recognition Letters 20 (1999) 199-206

9. Flusser, J., Zitová, B., Suk, T.: Invariant-based registration of rotated and blurred images. In Tammy, I.S., ed.: Proceedings IEEE 1999 International Geoscience and Remote Sensing Symposium, Los Alamitos, IEEE Computer Society (1999) $1262-1264$

10. Harikumar, G., Bresler, Y.: Perfect blind restoration of images blurred by multiple filters: Theory and efficient algorithms. IEEE Trans. Image Processing 8 (1999) 202-219

11. Pai, H.T., Bovik, A.: On eigenstructure-based direct multichannel blind image restoration. IEEE Trans. Image Processing 10 (2001) 1434-1446

12. Panci, G., Campisi, P., Colonnese, S., Scarano, G.: Multichannel blind image deconvolution using the bussgang algorithm: Spatial and multiresolution approaches. IEEE Trans. Image Processing 12 (2003) 1324-1337

13. Šroubek, F., Flusser, J.: Multichannel blind iterative image restoration. IEEE Trans. Image Processing 12 (2003) 1094-1106

14. Woods, N., Galatsanos, N., Katsaggelos, A.: EM-based simultaneous registration, restoration, and interpolation of super-resolved images. In: Image Processing, 2003. Proceedings. Volume 2. (2003) 303-306

15. Rav-Acha, A., Peleg, S.: Restoration of multiple images with motion blur in different directions. In: IEEE Workshop on Applications of Computer Vision (WACV). (2000) $22-27$

16. Sroubek, F., Flusser, J.: Shift-invariant multichannel blind restoration. In: Proceedings of the 3rd Int'l Symposium on Image and Signal Processing and Analysis, ISPA'03, Rome, IEEE (2003)

17. Aubert, G., Kornprobst, P.: Mathematical Problems in Image Processing. Springer Verlag, New York (2002) 